\title{
Design of Mine Safety Detection Alarm Based on Single-chip
}

\author{
Ma Ming Tao \\ Jilin College of Agricultural Science and Technology Information Engineering, Jilin 132101 \\ Jilin, China \\ e-mail: mmt800@126.com
}

\begin{abstract}
The design is based STC89C52 single-chip, using MQ-4 ammonia sensor and MQ-7 carbon monoxide sensor, while testing the ammonia and carbon monoxide, when the discovery of the detected gas concentration exceeds a certain concentration, the sensor voltage has changed. Through the LM393 comparator issue the corresponding signal to the single-chip STC89C52. Then single-chip received signal control stepper motor action, at the same time controlling the buzzer to alarm, reached early warning purposes.
\end{abstract}

Keywords- Single-chip STC89C52, Sensor, Stepper motor, Buzzer, Alarm

It contains large amounts of methane $(\mathrm{CH} 4)$ and other flammable gases in the coal mine, after causing huge economic losses accidents, endangering the lives of miners.
Currently the price of combustible gas alarm has been high on the market, and it need to periodically replace the sensor. Therefore, the design of a simple circuit, the relatively low price of a variety of devices to detect underground security alarm, In order to personal and property safety of the people and establish a reliable barrier is very necessary.

\section{SYSTEM PROGRAM}

According to the basic requirements of design, the system can be divided into three modules, the gas detection module, alarm module and stepper motor operating module (standby module, e.g., self-help function can be further oxygen). The overall system design circuit shown in Figure 1:

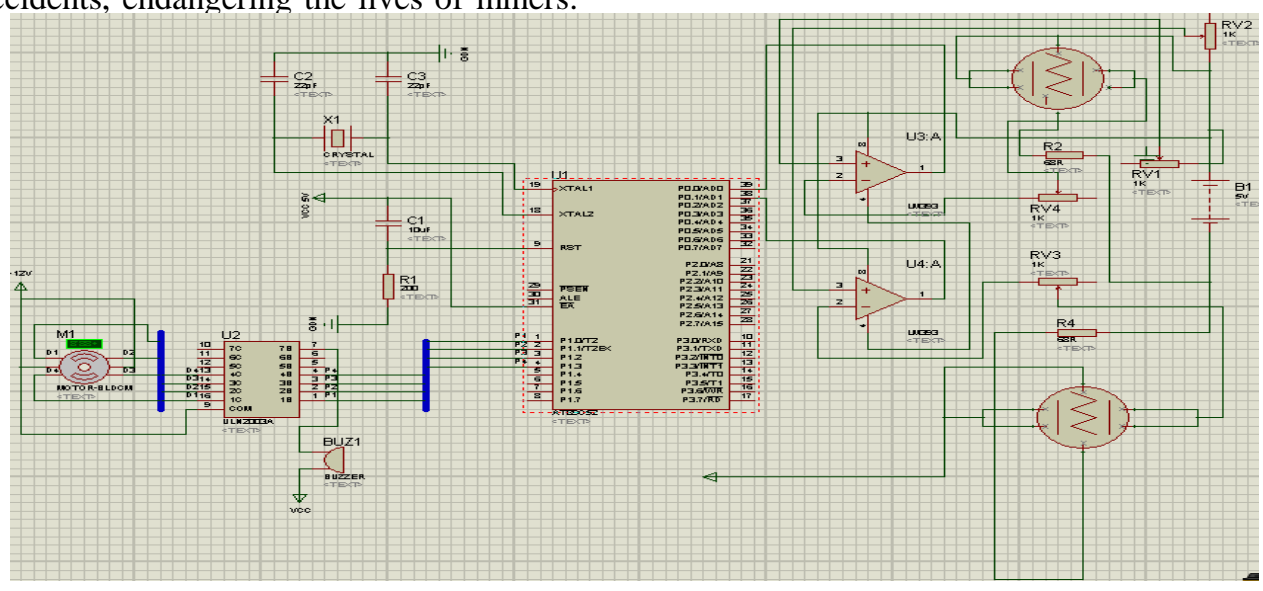

Figure 1.

System Circuit Diagram

\section{HARDWARE PART}

\section{A. the main control chip}

Using 52 series single-chip [1] STC89C52 for the control of core components. STC89C52 is STC produced a low-power, high-performance CMOS8 bit microcontroller with $8 \mathrm{~K}$ online system programmable Flash memory. STC89C52 using classical MCS-51 core [2], but it have a lot of improvements, making the traditional 51 single-chip has features not available. On a single-chip, 8-bit CPU has a smart and in-system programmable Flash, making STC89C52 provide highly flexible, ultra- effective solution for many embedded control applications.

\section{B. Gas Detection Circuit Design}

Gas sensor detection module consists of gas sensor [3] and comparator. Converted digital signal to the controller after processing the sensor by the controller to the digital processing is completed, the sensor portion using MQ-4 MQ7 and the test gas concentration, the sensor chip is used as a comparator with LM393P potentiometer sensitivity adjustment to complete gas collection and detection function.

When selecting a specific sensor, taking into account the operating voltage of the sensor, outside temperature adaptation, the sensitivity of the respective gas, whether the environment is suitable to withstand downhole environment, and the service life, so the choice of the sensor to ensure a certain degree of adaptation to the environment ability to be sensitive to a range greater than the range of the gas concentration of gas needed in our system, such products 
will have a more stable efficiency. Since the output signal of the sensor contains a certain amount of interference noise, it must be on the sensor output signal filtering [4], in the design of the filter circuit when using an ordinary small capacitor filter out high frequency interference, the use of large electrolytic capacitors to filter out low frequency interference. Electrical sensor output is relatively weak, usually millivolt, very weak is not easy to capture, and so the choice of comparator LM393, by varying the voltage effective value to achieve the objective detection circuit will be more sensitive to this, the efficiency will be higher. Design of the sensor driving circuit [5] shown in Figure 2:

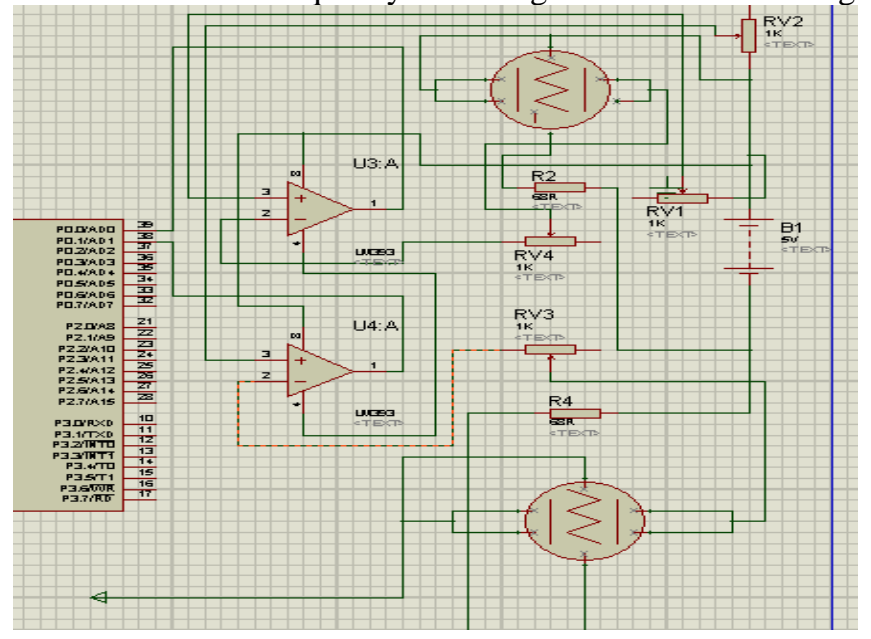

Figure 2. Sensor Drive Circuit Design

\section{Alarm Circuit Diagram}

Alarm section adopts the buzzer, alarm using ULN2003 chip drive, when the gas sensor detects gas concentration reaches a certain level, the single-chip microcontroller control chip ULN2003 to control the buzzer. Circuit shown in Figure 3:

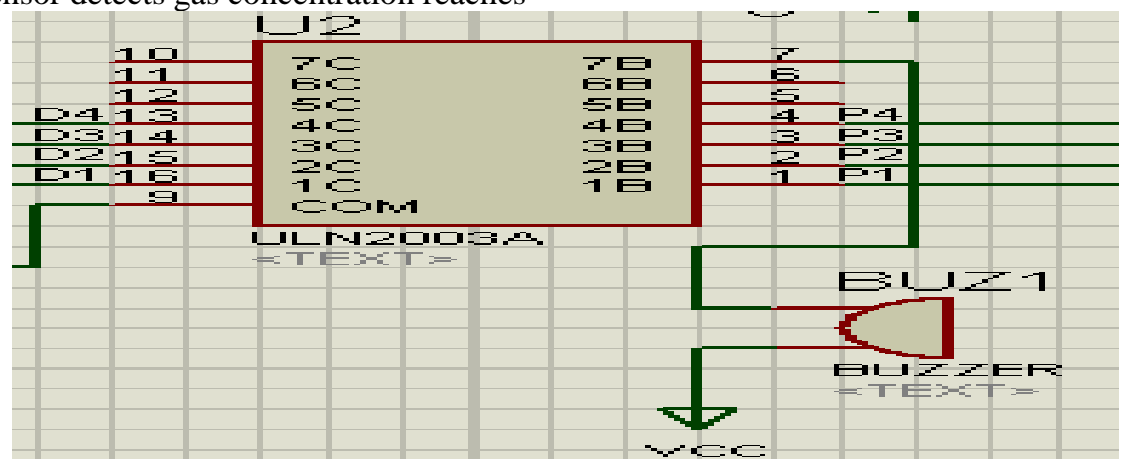

Figure 3.

Alarm Circuit

\section{Stepper Motor and Single-chip Circuit Connection Diagram}

Stepper motor alarm using ULN2003 chip driver, stepper motor circuit connection as shown in Figure 4: 


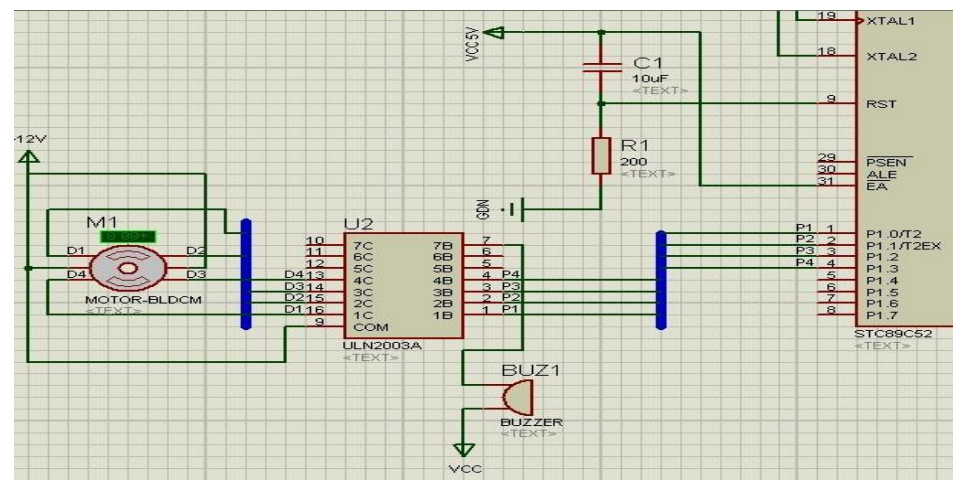

Figure 4.

Stepper Motor Circuit Connection Diagram

\section{SYSTEM SOFTWARE DESIGN}

\section{A. Main Program Flow Chart}

The main program flow chart shows the basic process system works, describes the basic flow of signals, played the role of a wizard. Main system flow chart shown in Figure 5:

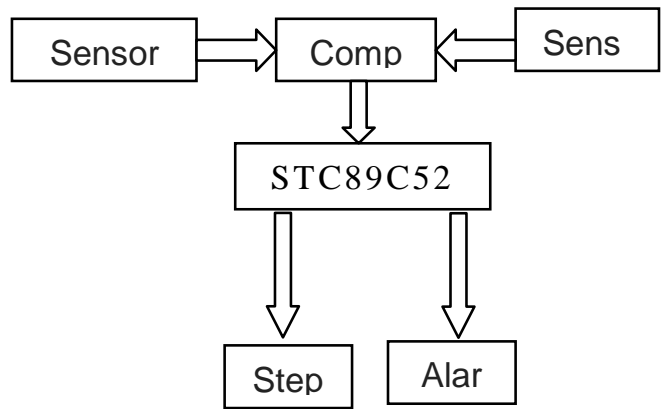

Figure 5. Main Program Flow Chart of System

\section{B. Signal Sampling and Routine Part of Stepper Motor Design}

Stepper motor subroutine mainly refers to the system starts running, the gas is passed over the sensor voltage value by the comparator compares the output signal and passed to the single-chip programming involved.

$$
\text { Main () }
$$

//unsigned int $\mathrm{i}=64 * 16$; / Stop switch 2 weeks

Speed $=3$; // Adjust the speed while(1)

$$
\text { Coil_A1 //Encounter Coil_A1 whit }
$$$$
\{\overline{\mathrm{A}} 1=1 ; \mathrm{B} 1=0 ; \mathrm{C} 1=0 ; \mathrm{D} 1=0 ;\} \text { instead }
$$

DelayMs(Speed); // Change this parameter can adjust motor speed,

// The smaller the number, the greater the speed, the smaller the moment

\section{Coil_B1}

DelayMs(Speed);

Coil_C1

DelayMs(Speed);

Coil_D1

DelayMs(Speed);<smiles>[AlH]I</smiles>

C. Sensor MQ-4 Subroutine Design

The main function

****************************************************

$* * * * * * * * * * * * * * * * * * * * * * * * 1$

void main()

\{

MQ-7

while(1) // Infinite loop

\{

LED $=1 ; / /$ Go Out P1.0 Lamp

if $($ DOUT $==0) / /$ When the concentration is higher than the set value, the function execution condition

$$
\{
$$

delay();//delay anti-interference

if $($ DOUT $==0) / /$ Determine the concentration is higher than the set value, the function execution condition

$$
\{
$$

LED=0; / LightenvP1.0 Lamp

D. Audible Alarm Subroutine

The main function

$\left\{\begin{array}{c}\text { main( }() \\ \text { unsigned int } \mathrm{i} ;\end{array}\right.$




$$
\begin{aligned}
& \text { while(1) } \\
& \{ \\
& \text { for }(\mathrm{i}=0 ; \mathrm{i}<200 ; \mathrm{i}++) \\
& \{ \\
& \text { DelayUs2x(200); } \\
& \mathrm{SPK}=\text { !SPK; } \\
& \text { \} }
\end{aligned}
$$

$\mathrm{SPK}=0 ; / /$ prevent damage to the speakers have been energized for $(\mathrm{i}=0 ; \mathrm{i}<200 ; \mathrm{i}++)$

$$
\text { DelayMs(1); }
$$$$
\text { \} }
$$$$
\text { \} }
$$

$$
/ *
$$

uS delay function, contains the input parameters unsigned char $\mathrm{t}$, no return value

unsigned char unsigned char variable is defined, its value range is

0 to 255 is used here crystal $12 \mathrm{M}$, precision delay use assembler, roughly delay

Length below $\mathrm{T}=\mathrm{tx} 2+5 \mathrm{uS}$

void DelayUs2x(unsigned char t)
while(--t);
\}

$\mathrm{mS}$ delay function, contains the input parameters unsigned char $t$, no return value

unsigned char unsigned char variable is defined, its value range is

0 255 is used here crystal $12 \mathrm{M}$, please use assembly precision delay

void DelayMs(unsigned char t)

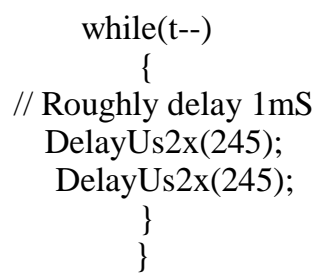

The single-chip as the core control system, use gas detection sensors and alarm systems to achieve the detection of dangerous gases underground, in order to protect the safety of mine workers . After testing, has been able to complete all of the features described.

\section{References}

[1] Wang Xingzhi, AT89 Series Single-chip Principle and Interface Technology [M].Beijing Aerospace Press,2006:45-65

[2] Zhang Yigang, MCS-51 Single-chip Application Design [M] Harbin: Harbin Institute of University Press .2003:142-169

[3] Jia Bonian, Yu Pu, Sensor Technology [M]. Southeast University Press, 2000:33-62

[4] Li Daohua, Li Ling, Zhu Yan, Sensor Circuit Analysis [M], Wuhan: Wuhan University Press, 2000:61-88

[5] Zhao Maota, Principles and Applications of Intelligent Instrument [M] Beijing: Electronic Industry Press, 2004:100-156 